\title{
CONTRIBUTION AU PROBLÈME DE LA FABRICATION DU "CASHCAVAL PENTELEU »
}

\author{
par
}

Ing. Carol TOMA et Ing. Ecaterina MELEGHI

Institut de Recherches Alimentaires. Bucarest.R. P. R.

Le Cashcaval fait partie de la catégorie des fromages ébouillantés. On le fabriquait déjà du temps des Romains. Son nom lui vient de l'italien "Cacio Cavallo" paree que, selon certains auteurs [1] on lui donnait (et on lui donne parfois encore) la forme d'une tête đe cheval ; selon d'autres, ce nom dériverait plutôt de la manière de transporter le "Cash » à dos de cheval.

De cette catégorie de fromages, font partie le produit italien Provolone et, chez nous, le Cashcaval de Dobroudja et le Cashcaval Penteleu, ce dernier étant spécifiquement roumain et fort apprécié par les consommateurs.

Le nom de Cashcaval Penteleu vient du mont Penteleu où sont apparus les premiers centres de fabrication. Longtemps, cette variété de fromage fut considérée, à juste titre, comme un des produits typiques de nos régions montagneuses.

Ces dernières années, par suite de l'augmentation de la consommation des produits laitiers et du développement de l'industrie laitière dans notre pays, la fabrication du Cashcaval Penteleu s'est aussi répandue dans les régions des collines et de la plaine. Néanmoins, la production garda son caractère artisanal, ce qui détermina une production non homogène et d'une qualité qui parfois laissait à désirer.

Dans le but de transformer cette production en lui donnant un caractère industriel et uniforme et une technologie améliorée, dans le but aussi, d'augmenter le rendement, une série de recherches ont été entreprises.

La littérature spécialisée présente peu de renseignements sur le cashcaval de Penteleu [2], [3], [4] : aucune interprétation scientifique de sa technologie, sauf quelques très insignifiants essais d'amélioration [5].

La manière dont les différentes phases du processus technologique peuvent influencer le rendement et la qualité du produit n'a pas été suffisamment étudiée.

La matière première qui sert à la fabrication du Cashcaval est le "Cash". On l'obtient du lait de brebis, du lait de vache, ainsi que de leur mélange.

Les principales phases du processus technologique sont : la réception de la matière première ("le Cash»), la maturation, 
l'ébouillantage du "Cash» avec de l'eau, sa mise en forme, sa salaison et sa maturation.

L'une des principales étapes du processus technologique est la détermination du moment optimum pour l'ébouillantage, moment où le degré de fermentation $d u$ "Cash » permet une fabrication en bonnes conditions.

Divers spécialistes intéressés à ce problème confirment nos dires.

Ainsi ont-ils montré que le Cashcaval ne pouvait s'obtenir d'un "Cash » insuffisamment ou excessivem ent fermenté, car, selon l'expression technique courante, la "liaison" serait incomplète et le produit friable [3].

N. Dimov [6] précise que d'un "Cash " insuffisamment maturé on obtient un Casheaval grumeux. Quant aủ "Cash " excessivement fermenté, on ne peut le pétrir et il donne, par conséquent, un produit peu satisfaisant.

Le même auteur affirme que, dans la production du Cashcaval, le moment optimum pour ébouillanter le "Cash" est la chose la plus difficile à établir ce fait a été confirmé par R. Marracino [7].

Cela se fait, aujourd'hui encore, de façon empirique, tenant compte de :

- la limpidité et la couleur du petit lait qui s'égoutte du "Cash ",

- le goût du "Cash » et son élasticité,

- la sonorité obtenue en le frappant arec la lame d'un couteau (épreuve de la percussion),

- sa consistance et son élasticité à l'ébouillantage (épreuve de l'ébouillantage).

De toutes ces épreuves, la plus concluante et la plus souvent employée est la dernière. Elle consiste en l'ébouillantage, dans une petite pelle en bois, d'un échantillon moyen de "Cash ».

C'est en fonction du comportement de cet échantillon de « Cash » soumis à l'ébouillantage, que le maître-artisan établit le moment propice pour commencer la fabrication du Cashcaval.

Toutes les méthodes indiquées par les spécialistes ou utilisées par les maîtres-artisans sont empiriques, donc subjectives, et ne peuvent pas constituer une base de travail pour une industrie moderne.

Ainsi, à la suite des analyses exécutées dans différents centres de fabrication, nous avons constaté que l'acidité du "Cash », considéré par les maîtres-artisans comme étant suffisamment maturé pour l'ébouillantage, allait de $150^{\circ}$ à $280^{\circ} \mathrm{T}$, limites beaucoup trop larges et prouvant la subjectivité des méthodes artisanales employées 
actuellement, méthodes dont les résultats sont nuisibles à la production.

Comme nous l'avons déjà mentionné, en ébouillantant un “Cash » insuffisamment maturé on obtient un produit qui ne correspond guère aux exigences de la qualité; d'autre part, l'ébouillantage d'un "Cash" ayant dépassé le degré de maturation normale, entraîne de grosses pertes de matière sèche dans l'eau d'ébouillantagè.

Les maîtres-artisans essaient de remédier à cette imprécision dans l'appréciation du degré de fermentation du « Cash » en variant sa durée d'ébouillantage.

Ainsi, dans le but d'éviter de grosses pertes de matière sèche dans l'eau d'ébouillantage, on réduit sensiblement la durée d'ébouillan tage du "Cash " excessivement maturé ; par ce fait, le rendement se trouve quelque peu amélioré, mais au détriment de la qualité du produit.

L'ébouillantage insuffisant du «Cash» suppose une destruction réduite de sà microflore; d'où le ballonnement du Cashcaval pendant la maturation (fig. 1 et 2 ).

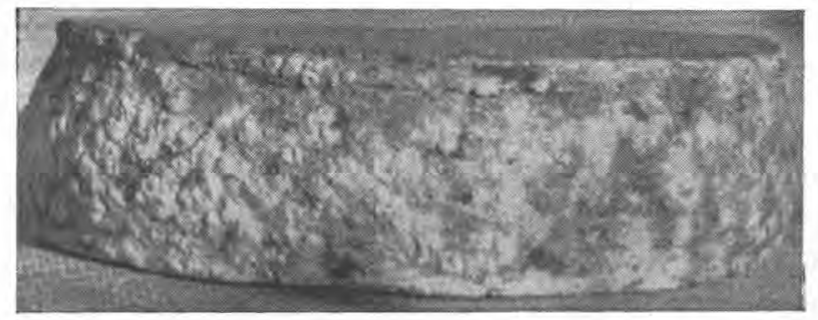

a) aspect extérieur

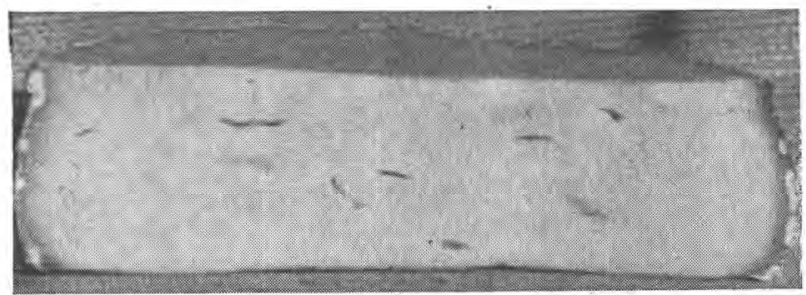

b) aspect intérieur

Fig. 1. - Casheaval Penteleu ayant subi une maturation normale.

Le manque de précision et la subjectivité de ces méthodes ont une influence considérable sur la qualité du produit, ainsi que sur le rendement. D'où la nécessité de les remplacer par des méthodes capables d'assurer l'exactitude, la rapidité et l'objectivité des déterminations. 
a) aspect extérieur

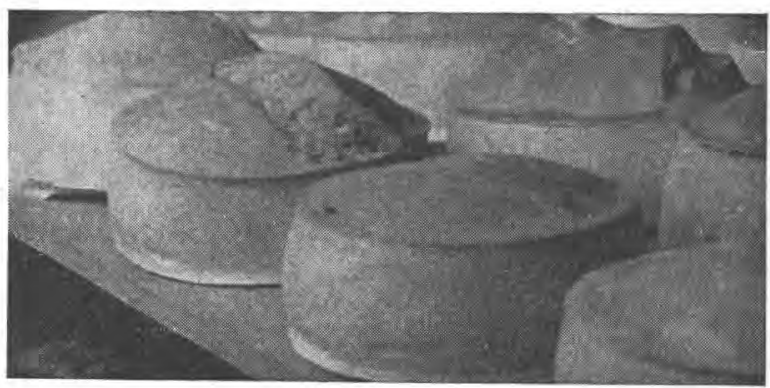

b) aspect intérieur

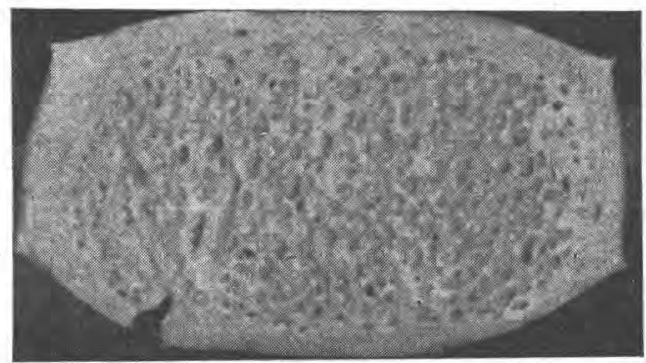

Fig. 2. - Cashcaval Penteleu ballonné.

Le but de nos recherches a été de :

- trouver une méthode objective et d'application facile, donnant des résultats concordants, susceptibles de servir à la détermination du moment optimum de l'ébouillantage du «Cash »,

- établir les limites de cet optimum,

- prouver l'intérêt qu'il y a à effectuer les ébouillantages dans les limites établies, afin que le processus technologique se déroule d'une manière satisfaisante.

\section{Recherches effectuées et résultats obtenus}

1. En vue d'établir le moment optimum d'ébouillantage du "Cash ", nous avons tout d'abord pris pour critérium d'appréciation son acidité totale exprimée en degrés Thörner.

Les déterminations ont été faites à l'aide de la méthode indiquée par Roeder [8].

Comme cette détermination n'a pas été suffisamment rapide par rapport aux conditions de travail dans la production, et que, d'autre part, les phénomènes fondamentaux de la maturation du "Cash " peuvent être mis en évidence de manière beaucoup plus satisfaisante en mesurant le $p H$ (opération rapide et commode), on 
a effectué parallèlement certaines déterminations du $p$ H à l'aide du papier Lyphan.

Nos essais en vue de déterminer le $p H$ à l'aide du potentiomètre qui fut mis à notre disposition, n'ont pas donné de résultats satisfaisants, les valeurs obtenues étant différentes entre elles.

En ce qui concerne la détermination colorimétrique du $p \mathrm{H}$, on a constaté que les meilleurs résultats étaient obtenus par l'introduction du papier indicateur dans une entaille fraîche faite dans le "Cash ».

2. En vue d'établir les limites du moment optimum d'ébouillantage, on a déterminé - pour le "Cash" de brebis eomme pour celui de vache - la variation de l'acidité en degrés Thörner et du $p$ H durant la maturation.

On a ébouillanté parallèlement divers échantillons de "Cash " en étudiant les pertes de matière sèche dans l'eau d'ébouillantage, ainsi que le comportement du "Cash " pendant I'ébouillantage.

La technique de travail a consisté dans l'appréciation du comportement pendant l'ébouillantage de certains échantillons parallèles de $100 \mathrm{~g}$ de "Cash ".

L'ébouillantage effectué au cours de toutes nos expériences a duré six minutes, durée moyenne des ébouillantages industriels.

Le comportement du "Cash" pendant l'ébouillantage a été déterminé en fonction de sa consistance plus ou moins filante et de son élasticité.

Les pertes de matière sèche dans l'eau d'ébouillantage homogénisée ont été déterminées gravimétriquement. Pour certaines expériences, les pertes ont été également déterminées par la différence entre la matière sèche du "Cash " employée et celle du "Cash " ébouillanté qui en fut obtenu. Les résultats ont exprimé des valeurs très rapprochées.

On trouvera dans les tableaux No 1 et $\mathrm{N}^{\circ} 2$ et les diagrammes des fig. $3,4,5$ et 6 les résultats d'un nombre certain d'expériences.

On constate qu'à mesure que l'acidité du "Cash " augmente et que, respectivement, son $p$ H diminue, on enregistre en général une légère augmentation des pertes de matière sèche pendant l'ébouillantage. Dans cette phase, le "Cash" a une consistance dure, gommeuse et se "lie » difficilement.

Dans la deuxième phase, les pertes diminuent jusqu'à un minimum et se maintiennent à un niveau relativement bas. Le "Cash" devient mou, filant et luisant et se "lie» facilement. Ce comportement correspond à un "Cash" ayant un degré de maturation optimum.

Dans la troisième phase, les pertes augmentent brusquement, 
TABLEAU I

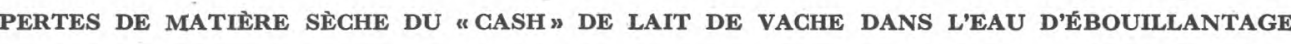

\begin{tabular}{|c|c|c|c|c|c|c|c|c|c|c|c|c|}
\hline \multirow{2}{*}{$\begin{array}{l}\text { Expé- } \\
\text { rience }\end{array}$} & \multirow{2}{*}{\multicolumn{2}{|c|}{$\begin{array}{l}\text { Ebouil- } \\
\text { lantage }\end{array}$}} & \multicolumn{2}{|c|}{ Cash cru } & \multirow{2}{*}{$\begin{array}{c}\text { Pertes } \\
\text { de matière } \\
\text { sèche } \\
\%\end{array}$} & \multirow{2}{*}{$\begin{array}{l}\text { Compor- } \\
\text { tement } \\
\text { du cash } \\
\text { à l'ébouil- } \\
\text { lantage }\end{array}$} & \multirow{2}{*}{$\begin{array}{l}\text { Expé- } \\
\text { rience }\end{array}$} & \multirow{2}{*}{$\begin{array}{l}\text { Ebouil- } \\
\text { lantage }\end{array}$} & \multicolumn{2}{|c|}{ Cash eru } & \multirow{2}{*}{$\begin{array}{c}\text { Pertes } \\
\text { de matière } \\
\text { sèche } \\
\%\end{array}$} & \multirow{2}{*}{$\begin{array}{c}\text { Compor- } \\
\text { tement } \\
\text { du cash } \\
\text { à l'ébouil } \\
\text { lantage }\end{array}$} \\
\hline & & & $\begin{array}{c}\text { (en degrés } \\
\mathrm{T} \text { ) }\end{array}$ & $p \mathbf{H}$ & & & & & $\begin{array}{c}\text { (en degrés } \\
\mathrm{T} \text { ) }\end{array}$ & $p \mathrm{H}$ & & \\
\hline \multirow{9}{*}{ I } & 1 & $\ldots$ & 170 & 5,3 & 13,38 & $*$ & & $1 \ldots \ldots$ & 185 & 5,2 & 14,5 & $*$ \\
\hline & 3 & $\ldots$ & 220 & 5,0 & 9,00 & $* * *$ & & $3 \ldots \ldots$ & 220 & 5,0 & 12 & $* * *$ \\
\hline & 4 & $\ldots$ & 230 & 5,0 & 9,70 & $* * *$ & & $4 \ldots \ldots$ & 235 & 4,9 & 13,2 & $* * *$ \\
\hline & 5 & $\ldots$ & 236 & 4,9 & 10,62 & $* * *$ & & $5 \quad \ldots \ldots$ & 240 & 4,8 & 13,2 & $* * *$ \\
\hline & 6 & $\cdots$ & 245 & 4,8 & 13,51 & $* * *$ & III & $6 \ldots \ldots$ & 243 & 4,8 & 13,4 & $* * *$ \\
\hline & 7 & $\ldots$ & 260 & 4,7 & 18,28 & $* *$ & & $7 \ldots$ & 250 & 4,7 & 17,2 & $* *$ \\
\hline & 8 & $\ldots$ & 266 & 4,7 & 19,85 & $* *$ & & $8 \ldots \ldots$ & 258 & 4,7 & 25 & $* *$ \\
\hline & 9 & $\ldots$ & 275 & 4,6 & 21,44 & $* *$ & & $9 \ldots \ldots$ & 270 & 4,6 & 27 & $* *$ \\
\hline & & $\cdots$ & 300 & 4,5 & 28,01 & $* *$ & & & & & & \\
\hline \multirow{8}{*}{ II } & 1 & $\ldots$ & 190 & 5,2 & 16,20 & $*$ & & & & & & \\
\hline & 2 & $\ldots$ & 210 & 5,1 & 18,82 & $*$ & & $1 \ldots \ldots$ & 158 & 5,4 & 11,1 & * \\
\hline & 3 & $\ldots$ & 225 & 5,0 & 17,30 & $* * *$ & & $2 \ldots \ldots$ & 190 & 5,2 & 12 & $*$ \\
\hline & 4 & $\cdots$ & 235 & 4,9 & 15,93 & $* * *$ & & $3, \ldots$ & 210 & 5,1 & 16 & $*$ \\
\hline & 5 & $\ldots$ & 242 & 4,8 & 18,67 & $* * *$ & IV & $4 \ldots \ldots$ & 225 & 5,0 & 14,3 & $* * *$ \\
\hline & 6 & $\ldots$ & 258 & 4,7 & 20,80 & $* *$ & & $5 \ldots \ldots$ & 240 & 4,8 & 13,6 & $* * *$ \\
\hline & 7 & $\ldots$ & 285 & 4,6 & 25,94 & $* *$ & & $6 \ldots$ & 250 & 4,7 & 19 & $* *$ \\
\hline & & $\ldots$ & 290 & 4,6 & 27,28 & $* *$ & & $7 \ldots \ldots$ & 252 & 4,7 & 20,6 & $* *$ \\
\hline
\end{tabular}

* Cash ayant une maturation insuffisante.

*** Cash ayant une maturation normale.

** Cash ayant une maturation excessive. 
TABLEAU. II

- PERTES DE MATIÈRE SĚCHE DU "GASH» DE LAIT, DE BREBIS DANS L'EAU D'ÉBOUILLANTAGE

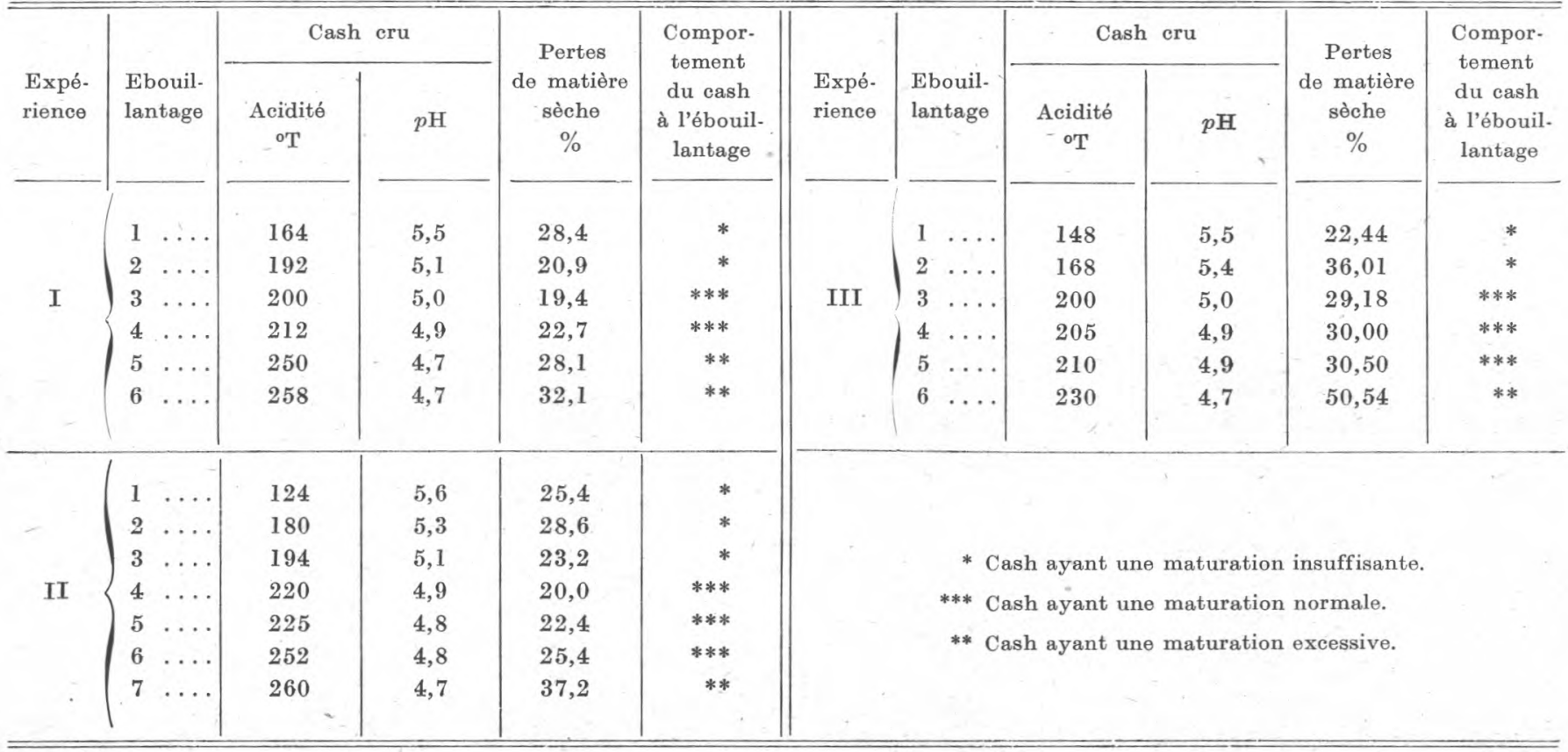




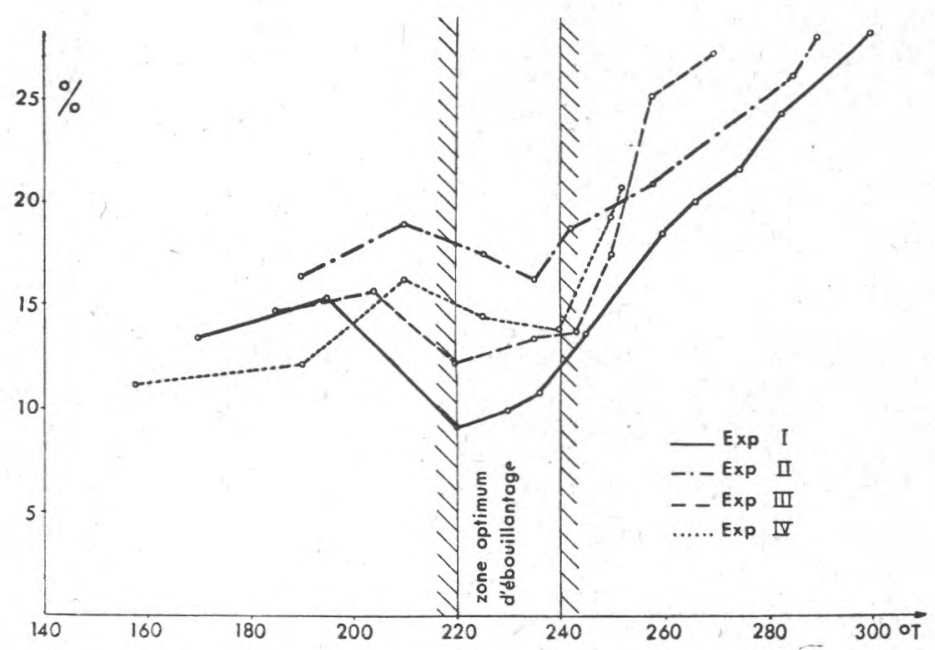

Fig. 3. - Variation des pertes de matière sèche du "cash" de lait de vache dans l'eau d'ébouillantage par rapport à l'acidité totale.

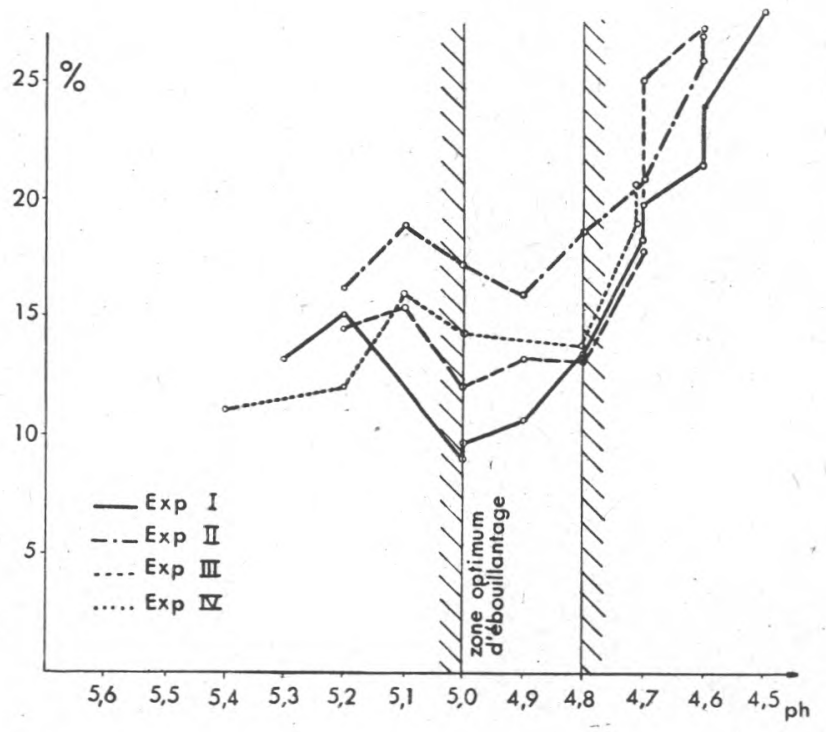

Fig. 4. - Variation des pertes de matière sèche du "cash" de lait de vache dans l'eau d'ébouillantage par rapport au $p H$. 


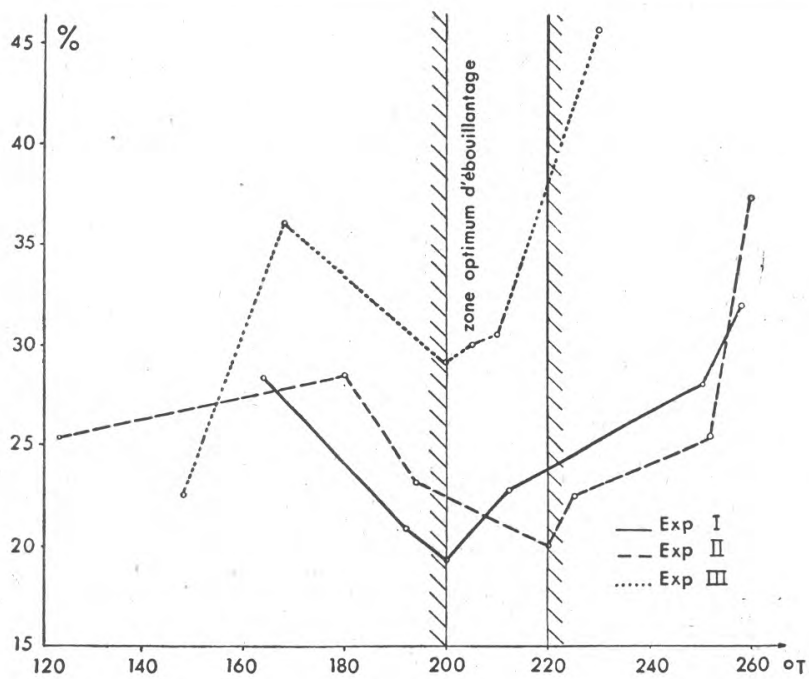

Fig. 5. - Variation des pertes de matière sèche du "cash " de lait de brebis dans l'eau d'ébouillantage, par rapport à l'acidité totale.

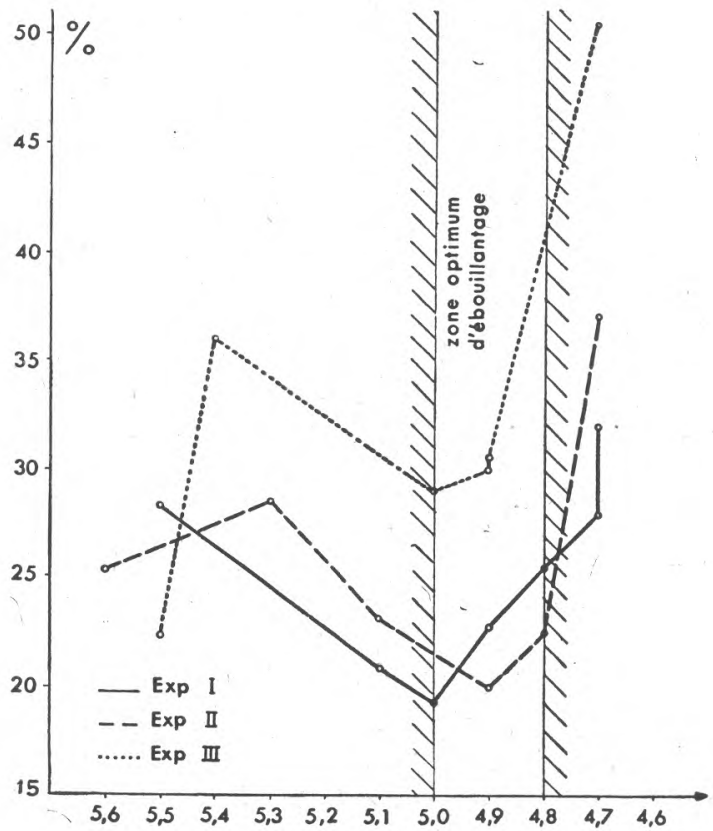

Fig. 6. - Variation des pertes de matière sèche du "cash " de lait de brebis dans l'eau d'ébouillantage, par rapport au $p H$. 
le "Cash» se disperse dans l'eau d'ébouillantage; il se "lie" difficilement, acquiert une structure granulaire et perd son luisant.

Nous avons donné le nom de "zone optimum d'ébouillantage» à la zone dans laquelle nous avons enregistré le minimum de pertes en matière sèche, pour l'échantillon dont la consistance et l'élasticité ont permis d'effectuer en bonnes conditions les opérations suivantes. Cette zone est comprise entre $p H 5$ et 4,8 , tant pour le "Cash " fabriqué du lait de vache, que pour celui préparé avec du lait de brebis.

Pour ce qui est des limites de l'acidité (exprimée en degrés Thörner) dans cette zone optimum, on a constaté qu'elles étaient comprises entre $220^{\circ}-240^{\circ} \mathrm{T}$ pour le "Cash" provenant du lait de vache, et entre $200^{\circ}-220^{\circ} \mathrm{T}$ pour le "Cash » de brebis.

Dans nos tableaux, le comportement du "Cash" pendant l'ébouillantage expérimental a été noté par les signes,+++ et +++ .

3. Les observations sur les fabrications industrielles du Cashcaval confirment les résultats expérimentaux.

La substitution aux méthodes artisanales actuelles (pour déterminer le moment optimum d'ébouillantage du "Cash") d'une méthode telle que celle indiquée par nous, ainsi que l'exéeution des ébouillantages dans la zone optimum établie, assureront l'amélioration du rendement et garantiront, du même coup, la qualité du produit.

Soulignons que la durée d'ébouillantage et la température de l'eau d'ébouillantage ont été maintenues constantes au cours des travaux expérimentaux. Il est à supposer que leur réduction - qui est possible dans la zone optimum - entraînera une plus importante diminution des pertes de matière sèche pendant l'ébouillantage.

Etant donné que la phase caractérisée par le moment optimum d'ébouillantage du "Cash" est incluse dans le processus technologique de fabrication de tous les fromages ébouillantés (Provolone, Cashcaval de Dobroudja etc.), nous considérons que les résultats que nous avons obtenus sont également applicables à la technologie de ces fromages.

\section{Conclusions}

1. Dans le processus technologique de fabrication du Cashcaval Penteleu, en particulier, et des fromages ébouillantés en général, la détermination du moment optimum d'ébouillantage constitue l'une des phases les plus importantes. La juste appréciation de ce moment est d'une importance particulière pour le rendement et la qualité du produit. 
2. Les méthodes employées jusqu'à présent pour déterminer ce moment optimum sont empiriques, donc subjectives; elles ont souvent pour conséquence une production de mauvaise qualité.

3. Grâce à des essais expérimentaux, on a pu constater qu'il était possible d'apprécier d'une manière objective, précise et rapide le degré de la maturation du "Cash », en déterminant son $p H$.

4. On a constaté que les pertes de matière sèche pendant l'ébouillantage du "Cash ", à des degrés différents de maturation, sont en fonction de son $p \mathrm{H}$.

5. Les pertes de matière sèche atteignent un minimum pour une valeur du $p \mathrm{H}$ comprise entre 5 et 4,8 , moment auquel les qualités plastiques du "Cash » sont les meilleures.

Cette zone de $p H$ comprise entre 5 et 4,8 a été dénommée "zone optimum d'ébouillantage ".

6. L'emploi de la méthode de travail indiquée et l'observance des limites de la zone optimum d'ébouillantage établis, permettront une amélioration du rendement et assureront en même temps la qualité du produit. Nous considérons que les résultats de nos travaux sur le Cashcaval Penteleu peuvent également s'appliquer à la technologie de tous les fromages ébouillantés.

N.B. - Les résultats de ces recherches ont été partiellement communiqués le 21. 11. 1958, à la session scientifique de l'Institut de recherches alimentaires de Bucarest.

\section{Summary}

The experiments conducted on a type of scalded cheese (Cashcaval Penteleu) revealed that it was possible to assess objectively, rapidly and precisely the most opportune moment to scald. An improvement in output and a better quality product resulted.

\section{BIBLIOGRAPHIE}

[1] M. StPkA, L'industrie du lait et des produits laitiers en Yougoslavie. Deutsche Molkerei Zeitung, 77, septembre 1956, no 38, 1307-1310.

[2] N. Odarsky. La préparation des fromages. Bucarest. 1945. 120 p., 50-53.

[3] M. Albu et V. Argesiu. La technologie du lait et des produits laitiers. Bucarest. 1954. Ed. Technique, 324 p., 196-199.

[4] C. Stoian. Compte rendu concernant la fabrication du Cashcaval, dans la R. P. R. Bucarest. 1957.

[5] H. Weigmann. Handbuch der Praktischen Käserei. 1933. Ed. 4, 680692. 